\title{
X-ray as Irradiation Alternative for K562 Feeder Cell Inactivation in Human Natural Killer Cell Expansion
}

\author{
GWANG HO KIM ${ }^{1 *}$, HOANG-NGUYEN DANG ${ }^{1 *}$, MINH-TRANG THI PHAN ${ }^{2,3 *}$, \\ SOON HO KWEON ${ }^{4}$, SEJONG CHUN ${ }^{5}$ and DUCK $\mathrm{CHO}^{1,3,4,6}$
${ }^{1}$ Department of Health Sciences and Technology, Samsung Advanced Institute for Health Sciences and Technology, Sungkyunkwan University, Seoul, Republic of Korea;
${ }^{2}$ Department of Mechanical Engineering at POSTECH, Gyeongbuk, Republic of Korea;
${ }^{3}$ Department of Laboratory Medicine and Genetics, Samsung Medical Center,
Sungkyunkwan University School of Medicine, Seoul, Republic of Korea;
${ }^{4}$ School of Interdisciplinary Bioscience and Bioengineering (I-Bio), POSTECH, Pohang, Republic of Korea;
${ }^{5}$ Department of Laboratory Medicine, Chonnam National University Hospital,
Chonnam National University Medical School, Gwangju, Republic of Korea;
${ }^{6}$ Stem Cell \& Regenerative Medicine Institute, Samsung Medical Center, Seoul, Republic of Korea

\begin{abstract}
Background/Aim: $\gamma$-Irradiation has been proven to be the most effective method to inactivate K562 cells, but $\gamma$-irradiators are not available in some institutes. This study was designed to compare the effects of $X$-ray and $\gamma$-irradiation on K562 cells in natural killer (NK) cell expansion. Materials and Methods: To expand NK cells, isolated peripheral blood mononuclear cells (PBMCs) were co-cultured with $\gamma$-irradiated or X-ray-treated K562 cells plus IL-2 and IL-15. Characteristics of expanded NK cells were identified by flow cytometry. Results: $N K$ cell expansion rate tended be to lower in the X-ray-treated group (68.9 \pm 32.6$)$ than the $\gamma$-irradiated group (78 \pm 28.7$)$, but the difference was not significant $(p=0.39)$. Furthermore, $N K$ cell functions or receptor expression were similar in the two groups. Conclusion: Our results suggest that $X$-ray treatment can be used as an alternative to $\gamma$-irradiation for K562 cells inactivation in human $N K$ cell expansion.
\end{abstract}

Natural killer (NK) cells are CD3-CD56+ and belong to a lymphocyte subpopulation capable of recognizing and killing cancer cells without prior immunization. It is well-known that NK cells (i.e., NK-92) are potential tools for cancer

*These Authors contributed equally to this work.

Correspondence to: Duck Cho, MD, Ph.D., Department of Laboratory Medicine \& Genetics, Samsung Medical Center, Sungkyunkwan University School of Medicine, 81 Irwon-dong, Gangnam-gu, Seoul, 06351, Republic of Korea. Tel: +82 234102403, Fax: +82 234102719, e-mail: duck.cho@skku.edu

Key Words: X-ray, irradiation, K562, feeder cell, inactivation. immunotherapy $(1,2)$. However, the application of NK cells in the clinical field would be a challenge due to the small portion and number of NK cells in peripheral blood mononuclear cells (PBMCs) (3). Therefore, expanding NK cells to obtain large numbers would be advantageous.

It has been reported that NK cells can be expanded upon cytokine activation, such as interleukin (IL)-2, and by interacting with accessory cells. Some NK cell expansion methods have been developed based on co-culturing PBMCs with cancer cell lines as feeder cells, including K562, Epstein-Barr virus-transformed lymphoblastoid cell line (EBV-LCL), RPMI 1866, and the Wilms tumor cell line (HFWT) (4-9). Among the cell lines tested as feeder cells, K562 has been reported as the most commonly utilized cell line for NK cell expansion. However, K562 feeder cells should be inactivated before co-culture to avoid the overgrowth of K562 cells. Gamma $(\gamma)$-irradiation has been reported as a commonly used method because of its effectiveness compared to other methods, such as mitomycin $\mathrm{C}$ treatment. However, $\gamma$-irradiators are not always available in certain institutes. Therefore, mitomycin $\mathrm{C}$ is alternatively used to inactivate feeder cells in some groups. However, the expansion rate was remarkably low when mitomycin $\mathrm{C}$ treatment was used instead of irradiation (10). Therefore, there is a need to study alternative methods.

Few studies have been conducted on X-ray treatment of feeder cells for NK cell expansion. Therefore, the present study was designed to compare the effects of X-ray treatment and $\gamma$-irradiation on K562 feeder cells used for NK cell expansion. X-ray treatment is suggested as an alternative method for non-replicating feeder cells for NK cell expansion. 


\section{Materials and Methods}

Cell lines. K562 (human myelogenous leukemia cell line) and Raji (human Burkitt's lymphoma cell line) were obtained from the American Type Culture Collection (ATCC, Manassas, VA, USA). These cell lines were maintained in RPMI-1640 medium supplemented with $10 \%$ fetal bovine serum FBS (Gibco, USA), 100 $\mathrm{U} / \mathrm{ml}$ penicillin, and $100 \mu \mathrm{g} / \mathrm{ml}$ streptomycin (Lonza, Walkersville, $\mathrm{MD}, \mathrm{USA})$ at $37^{\circ} \mathrm{C}$ in a humidified incubator containing $5 \% \mathrm{CO}_{2}$.

Cytokine and antibodies. Recombinant human IL-2 and IL-15 (PeproTech, Rocky Hill, NJ, USA) were used to expand NK cells from PBMCs. AmCyan-conjugated CD45 (Cytognos, Salamanca, Spain), PE-Cy7-conjugated CD56, APC-Cy7-conjugated CD3, Pacific blue-conjugated CD16, fluorescein isothiocyanate (FITC)conjugated CD57, APC-conjugated NKG2A, PE-conjugated NKG2C (R\&D System, Minneapolis, MN, USA), PerCP-conjugated CD8a, Pacific blue-conjugated NKp46, PE-conjugated NKp30, FITC-conjugated CD62L, PerCP-conjugated NKG2D (eBioscience, San Diego, CA, USA), and APC-conjugated DNAM-1 (BD Biosciences, San Jose, CA, USA) antibodies were used to examine purity and the surface expression of NK cell receptors. (PE)conjugated antibody to human HLA-ABC (BD Biosciences), MICA, MICB, ULBP1, ULBP2, ULBP3 antibodies and isotype controls (R\&D System) were used to compare marker expression on K562 cells following $4 \mathrm{~h}$ and $24 \mathrm{~h}$ treatment with either X-ray or $\gamma$ irradiation.

Expansion of NK cells from PBMCs. Our Institutional Review Board (No. SMC 2017-11-051) approved this study and no data were used for personal identification of human PBMCs. PBMCs were isolated from healthy adult donors using density-gradient centrifugation with Ficoll-Hypaque (d=1.077, Lymphoprep ${ }^{\mathrm{TM}}$; Axis-Shield, Oslo, Norway) and washed twice with phosphate-buffered saline (PBS). The expansion process was performed as previously described (11). Freshly isolated PBMCs $\left(3 \times 10^{6}\right.$ cells $)$ were co-cultured with $50 \mathrm{~Gy}-$ $\gamma$-irradiated K562 cells or $50 \mathrm{~Gy}-\mathrm{X}$ ray-irradiated K562 cells $\left(0.5 \times 10^{6}\right.$ cells $)$ in a 24 -well tissue culture plate in the presence of 10 $\mathrm{U} / \mathrm{ml}$ recombinant human IL-2 in complete RPMI-1640 medium (RPMI-1640 supplemented with 10\% FBS, 4 mM-glutamine, $100 \mathrm{U} / \mathrm{ml}$ penicillin, and $100 \mu \mathrm{g} / \mathrm{ml}$ streptomycin). The medium was replaced every 2-3 days with fresh medium containing $10 \mathrm{IU} / \mathrm{ml}$ human IL-2. After 7 days of culturing, the concentration of IL-2 was increased to $100 \mathrm{IU} / \mathrm{ml}$, and $10 \mathrm{IU} / \mathrm{ml} \mathrm{IL}-15$ was added to the medium. The medium was replaced every $2-3$ days.

NK cell receptors and K562 ligands analysis. The surface expression of NK cell markers after the expansion period was analyzed by flow cytometry. Briefly, $2 \times 10^{5}$ cells were washed with $1 \mathrm{X}$ PBS and 1\% FBS and stained with an appropriate combination of fluorochrome-conjugated monoclonal antibodies (CD45, CD56, CD3, CD16, NKp46, NKp30, CD8a, NKG2D, NKG2A, NKG2C, and DNAM-1) for $15 \mathrm{~min}$. To detect NKG2D ligands on the cell surfaces following K562 treatment with either X-ray or $\gamma$ irradiation, K562 cells $\left(1 \times 10^{5}\right)$ were stained for HLA-ABC, MICA, MICB, ULBP1, ULBP2, and ULBP3 as well as appropriate isotype controls. The cells were washed twice with $1 \mathrm{X}$ PBS and $1 \%$ FBS and fixed in $1 \mathrm{X}$ PBS and $1 \%$ formaldehyde before acquisition on the FACS Cano II (BD Biosciences). Data were analyzed by Kaluza (Beckman Coulter, Brea, CA, USA).
Evaluation of viability of K562 cells after X-ray or $\gamma$-irradiation. $\mathrm{X}$-ray treated and $\gamma$-irradiated K562 cells were maintained in culture in complete RPMI-1640 medium without PBMCs for 10 days to evaluate apoptosis induction. Cell viability was examined on day 0 , 3, 7, and 10 using the FITC Annexin V/Dead Cell Apoptosis Kit (Invitrogen, Eugene, OR, USA), according to the manufacturer's instructions. Briefly, irradiated K562 cells were washed in cold PBS. Cells were suspended in $100 \mathrm{ml}$ of $1 \mathrm{X}$ annexin-binding buffer containing $5 \mu \mathrm{l}$ of FITC annexin $\mathrm{V}$ and $1 \mu \mathrm{l}$ of the propidium iodide (PI) solution. The cells were incubated at room temperature for 15 min; subsequently, $400 \mu \mathrm{l}$ of $1 \mathrm{X}$ annexin-binding buffer was added to the samples for flow cytometry.

The percentage of non-replicating $K 562$ feeder cells survived following $X$-ray treatment or $\gamma$-irradiation during NK cell expansion. To calculate the percentage of non-replicating K562 feeder cells survived following X-ray treatment or $\gamma$-irradiation during NK cell expansion, X-ray treated and $\gamma$-irradiated K562 cells were stained with $5 \mu \mathrm{M}$ carboxyfluorescein diacetate succinimidyl ester (CFSE) prior to co-culturing with PBMCs. CFSE-positive K562 cells on day $0,3,7$, and 10 were identified and counted by flow cytometry.

Flow cytometric cytotoxicity assay and antibody-dependent cellmediated cytotoxicity (ADCC) assay. For the cytotoxicity assay, $1 \mu \mathrm{M}$ CFSE-stained K562 were incubated in a 96-well U-bottom plate with expanded NK cells at effector-to-target (E:T) ratios 2:1; $1: 1$, and $0.5: 1$ for $4 \mathrm{~h}$ at $37^{\circ} \mathrm{C}$ in a $5 \% \mathrm{CO}_{2}$ incubator. To perform the ADCC assay, Raji cells were incubated with $1 \mu \mathrm{g} / \mathrm{ml}$ of rituximab for $4 \mathrm{~h}$ and then stained with $1 \mu \mathrm{M}$ CFSE. The CFSEstained rituximab-coated Raji cells were also co-incubated with expanded NK cells using the same E:T ratios described above for the cytotoxicity assay. The mixed cells were transferred to FACS tubes after incubation. Before acquisition (5-10 min), $1 \mu 1$ of 1 $\mathrm{mg} / \mathrm{ml}$ PI (Invitrogen) was added to each tube. The cells were acquired on a FACS Canto II and analyzed using Kaluza software.

Statistics. Statistical evaluation was performed using the Prism 5 software (GraphPad Software, San Diego, CA, USA). The MannWhitney test (non-parametric) was applied to analyze differences between groups in term of purity, fold expansion, cytotoxicity, and ADCC of expanded NK cells; viability and the frequency of K562 cells, with a $p$-value $<0.05$ considered as statistically significant.

\section{Results}

NK cell expansion rate and purity. Using two feeder cell groups, the purity and expansion rate of NK cells was compared from PBMCs isolated from six healthy donors. The mean NK cell purity was similar in X-ray treated group $(69.7 \pm 10.1 \%)$ and $\gamma$-irradiated group $(70.1 \pm 9.89 \%)$ on day 14 of culture $(p=0.87)$. The 14-day NK cell expansion rate tended to be lower in the X-ray treated group $(68.9 \pm 32.6)$ than in the $\gamma$-irradiated group (78 \pm 28.7$)$, but a significant difference was not detected between the two groups $(p=0.39)$ (Figure 1).

Expression of NK cell receptors, Cytotoxicity, and ADCC of expanded NK cells. Expression of NK cell receptors (CD16, 
CD57, NKG2A, NKG2C, CD8a, NKp30, NKp46, CD62L, NKG2D, and DNAM-1) on expanded NK cells did not differ significantly between the two groups, in terms of both percentage of cells expressing a specific receptor and the relative expression [mean fluorescence intensity (MFI)] of each receptor analyzed (Figure 2). Direct cytotoxic effects of expanded NK cells against K562 cells and ADCC against rituximab-coated Raji cells were compared. At an E:T ratio of 2:1, 1:1, or $0.5: 1$, no significant difference in cytotoxicity was detected between the $\mathrm{X}$-ray and $\gamma$-irradiation groups ( $p>0.05$ in all ratios) (Figure 3$)$.

NKG2D ligand expression, viability, and percentage of survived non-replicating $X$-ray treated and $\gamma$-irradiated K562 feeder cells. The cell surface expression of NKG2D ligand in X-ray treated and $\gamma$-irradiated K562 feeder cells was examined by flow cytometry. The $\mathrm{X}$-ray and $\gamma$-irradiated K562 cells had similar levels of HLA-ABC, ULBP1, ULBP2, and ULBP3 compared to non-treated K562 cells, whereas MICA and MICB expression increased $4 \mathrm{~h}$ and 24 $\mathrm{h}$ after treatment. However, no significant difference in relative mean fluorescence intensity (rMFI) of MICA $(p=0.2)$ or MICB $(p=0.8)$ was found in X-ray and $\gamma$ irradiated K562 cells (Figure 4).

Viability of K562 feeder cells after treatment with X-ray or $\gamma$-irradiation was examined. Both X-ray treated and $\gamma$-irradiated K562 cells did not proliferate, only $16.04 \pm 0.85 \%$ and $13.39 \pm 0.67 \%$ of the K562 cells survived at day 10 of culture, respectively. Further, a significant difference in viability of $\mathrm{X}$ ray treated and $\gamma$-irradiated K562 cells was not found by flow cytometric apoptosis assay $(p=0.1)$ (Figure $5 \mathrm{~A}$ ).

The percentage of survived X-ray treated or $\gamma$-irradiated K562 cells at varying time points during NK cell expansion was also compared. No significant difference was observed in the percentage of CFSE-positive feeder cells at culture days $0,3,7$, and 10 during NK cell expansion $(p=0.1 ; p=01$; $p=0.7 ; p=0.4$, respectively). Only $0.48 \pm 0.11 \%$ and $0.55 \pm 0.06$ $\%$ of CFSE-positive K562 cells remained at day 10 of culture in X-ray treated and $\gamma$-irradiated groups (Figure 5B).

\section{Discussion}

This study demonstrated for the first time that X-ray treatment can be used as an alternative to $\gamma$-irradiation in order to inactivate K562 feeder cells to be used in human NK cell expansion.

When a $\gamma$-irradiator was not available, MMC was alternatively used to inactivate feeder cells. However, unsatisfactory fold expansion of NK cells from CD3+ depleted lymphocytes was reported when using MMC-treated K562 feeder cells; approximately 20 -fold expansion of NK cells was reached at day 15 of culture (10). Another group observed that expansion of human B cells was 100-times lower in the co-culture system with MMC-treated feeder cells than $\gamma$-irradiated feeder cells, and that their metabolic activity, viability, and ligand expression were significantly decreased (12). Therefore, these data suggest that MMC-treated feeder cells cannot support efficiently the growth of target cells (i.e. NK cells, B cells). As such, X-ray was investigated as an alternative to $\gamma$-irradiation rather than MMC due to comparable properties of $\gamma$-irradiation and X-rays.

In this study, the purity and fold expansion of NK cells and the functional characteristics of NK cells expanded on $\mathrm{X}$-ray treated or $\gamma$-irradiated K562 feeder cells were compared. The expansion rate in the $\mathrm{X}$-ray group showed a tendency to be lower than the $\gamma$-irradiation group, significant differences in purity and fold expansion of NK cells were not detected between the two groups. Because the biochemical effects of X-ray and $\gamma$-irradiation on K562 cells have not been well characterized and compared, the small but insignificant difference observed between the two groups cannot be explained. Subsequently, the functional characteristics of NK cells expanded on X-ray treated and $\gamma$ irradiated K562 feeder cells were compared with respect to their cytotoxicity against the K562 cells, ADCC against the rituximab-coated Raji cells, and NK cell receptor expression (CD16, NKG2A, NKG2C, CD57, CD8a, NKp30, NKp46, CD62L, DNAM-1, and NKG2D). No significant differences were found between the two groups. These data suggest that the biochemical effects of $\gamma$-irradiation and X-ray treatment of K562 feeder cells might be similar regarding the functional characteristics of expanded NK cells.

During the NK cell expansion, proliferation of K562 feeder cells should be inhibited, such that the feeder cells eventually disappear or be reduced. Therefore, viability after treatment by X-ray or $\gamma$-irradiation and the percentage of survived K562 feeder cells during NK cell expansion were also studied. Proliferation of K562 feeder cells in the two groups was blocked, and the percentage of survived K562 cells during NK cell expansion was minimal (less than $0.6 \%$ of K562 cells remained at day 10 of culture in both groups). However, compared with previous studies by Lapteva et al. (13) or Baek et al. (14), the percentage of K562 feeder cells within the population of $\mathrm{NK}$ cells in our study was considerably high, and did not satisfy the release criterion of $<0.1 \%$ percentage of feeder cells within the NK cell population. This difference can derive from the low-dose ionizing radiation (50 Gy) we selected to inactivate K562 cells, which might be acceptable for the comparison of purity, fold expansion, and function of expanded NK cells in the two groups. However, with respect to the percentage of K562 feeder cells remained within the NK cell population, further study is required, especially when it is considered for clinical applications. The feeder cells must be lethally irradiated (at least $100 \mathrm{~Gy}$ ) prior to use in culture to avoid the risk of infusing viable feeder cells. 

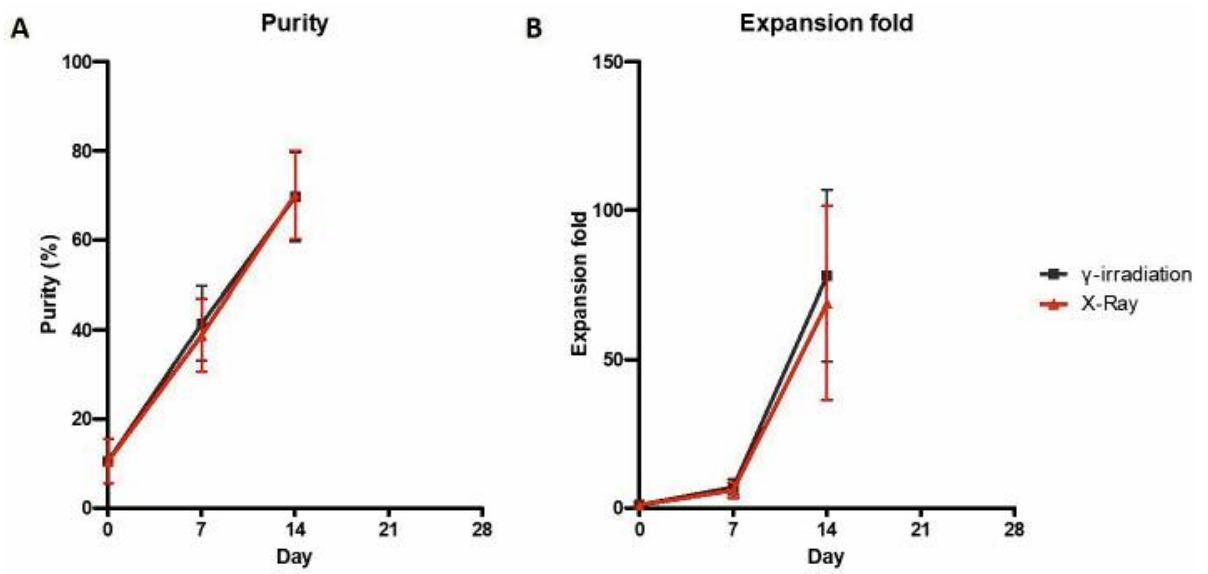

Figure 1. The purity and fold expansion of expanded NK cells co-culture with X-ray-treated K562 feeder cells and $\gamma$-irradiated K562 feeder cells. (A) The purity of expanded NK cells was determined by flow cytometry with the use of PE-Cy7-conjugated CD56, APC-Cy7 conjugated CD3. Mean $N K$ cell purities in two groups were not significantly different on day 0 , day 7, and day 14 of culture ( $p>0.05)$. (B) Mean fold expansion of NK cells in the X-ray treated group versus the $\gamma$-irradiatation-treated group did not significantly differ after two weeks of culture ( $p>0.05)$.
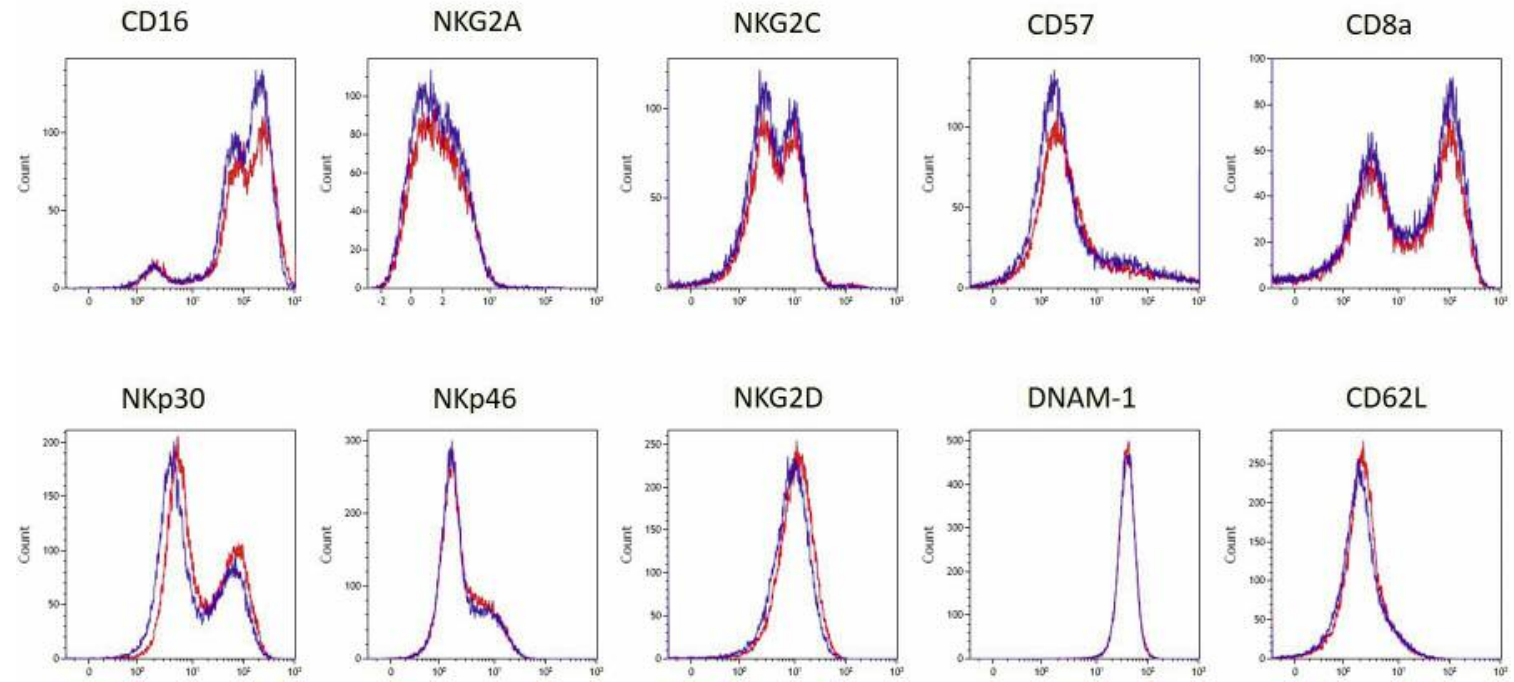

Figure 2. Expression of surface receptors on expanded NK cells using X-ray and $\gamma$-irradiation K562 feeder cells. Expanded NK cells on day 14 were assayed for CD16, CD57, NKG2A, NKG2C, CD8a, NKp46, NKp30, NKp46, CD62L, NKG2D and DNAM-1. Representative histograms are shown in NK cell subset, overlaying $\gamma$-irradiation group (blue) and X-ray group (red). NK cells expanded with X-ray-treated K562 feeder cells have similar expression of CD16, CD57, NKG2A, NKG2C, CD8a, NKp46, NKp30, NKp46, CD62L, NKG2D and DNAM-1 compared to those of $\gamma$-irradiated K562 feeder cells $(n=6)$.

Radiation has been known to have immune-modulating activities by triggering NK cell receptors through NKG2D ligands expressed in tumor cells $(15,16)$. In this study, the expression of NKG2D ligands (ULBP1, ULBP2, and ULBP3) did not change, whereas expression of MICA/B was slightly increased after $24 \mathrm{~h}$ treatment by X-ray or $\gamma$-irradiation.

Taken together, although the purity and fold expansion of NK cells in the X-ray group showed a tendency to be lower than the $\gamma$-irradiation group, no significant difference was detected between the two groups. Characteristics of NK cells expanded on the two groups of feeder cells (X-ray vs. $\gamma$-irradiation) did not significantly differ. Our results suggested that $\mathrm{X}$-ray treatment can be an alternative to irradiation to inactivate K562 feeder cells to be used for human natural killer cell expansion, especially since $\gamma$-irradiators are not available at some institutes. 

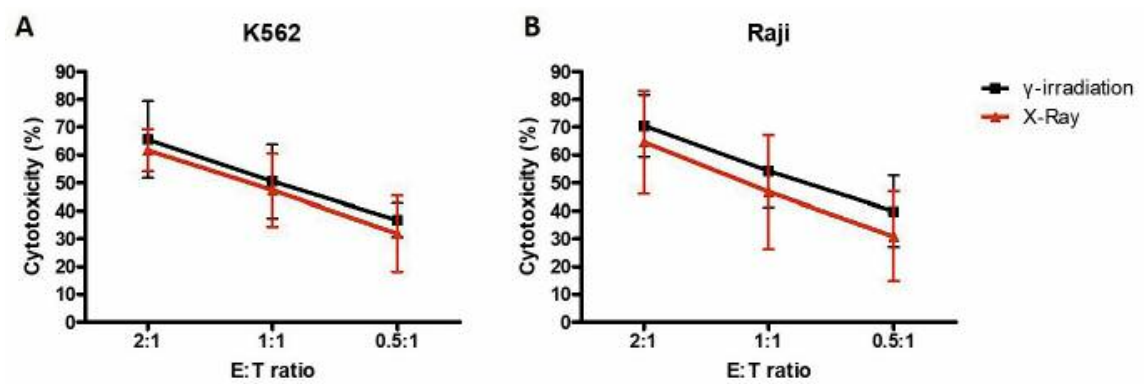

Figure 3. Cytotoxicity of NK cells expanded using X-ray and $\gamma$-irradiation feeder cells against K562 and ADCC against Raji. Cytotoxicity of expanded NK cells on day 14 toward K562 cells (A) and ADCC of expanded NK cells against rituximab-coated Raji cells (B) were measured by CFSE-based flow cytometry assay at E:T ratio of 2:1, 1:1, 0.5:1. The results presented are means $\pm S D$ of samples from six donors. All samples were tested in triplicate.
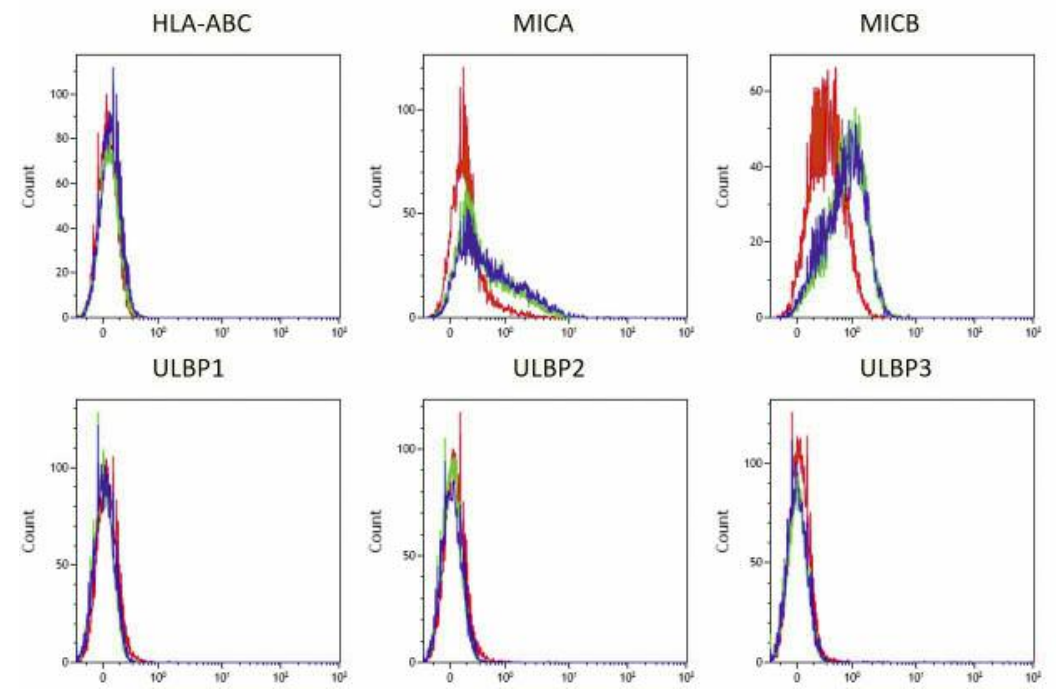

Figure 4. Flow cytometric characterization of NKG2D ligand expression on K562 cells after 24 h treatment with X-ray or $\gamma$-irradiation. Representative flow cytometry histograms of three independent experiments are shown (red: fresh K562, green: $\gamma$-irradiated K562 after 24 h, blue: $X$-ray treated $K 562$ after $24 h$ ). Expression of $H L A-A B C, U L B P 1, U L B P 2, U L B P 3$ were not changed after irradiation; expression of MICA and $M I C B$ were up-regulated by treatment with $X$-ray or $\gamma$-irradiation, but similar rMFI of MICA $(p=0.2)$ or MICB ( $p=0.8)$ was found in $X$-ray and $\gamma$-irradiated K562 cells.

A

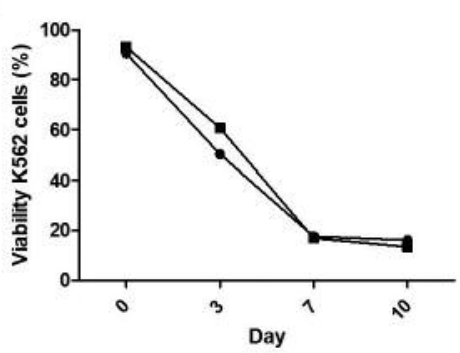

B

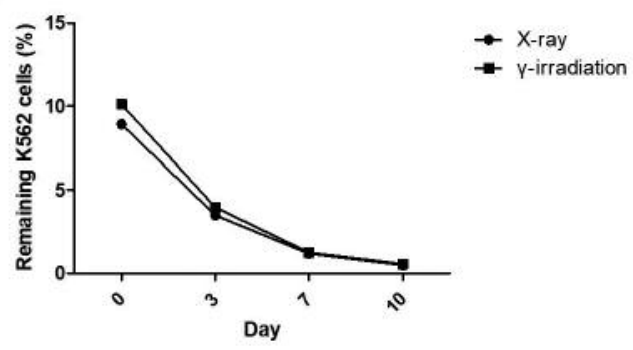

Figure 5. Viability of X-ray treated and $\gamma$-irradiation-treated $K 562$ feeder cells and the frequency of K562 feeder cells remained in the expanded NK cell system. (A) Viability of K562 feeder cells were examined with apoptosis kit by flow cytometry. X-ray and $\gamma$-irradiation treated K562 feeder cells did not proliferate after culture. (B) The percentage of $K 562$ feeder cells remained following $X$-ray treatment or $\gamma$-irradiation in the expanded NK cells system were analyzed by measuring CFSE-stained K562 cells alone. Non-significant difference was observed in X-ray and $\gamma$-irradiation group. Summary data from three donors, and all samples were tested in triplicate. 


\section{Acknowledgements}

This study was supported by Basic Science Research Program through the National Research Foundation of Korea (NRF) funded by the Ministry of Education, Science and Technology (2015R1D1A1 A09058740 and 2018R1A2B6006200) and a Grant from the Korea Research Institute of Bioscience and Biotechnology (KGM4941814).

\section{References}

1 Malmberg KJ, Bryceson YT, Carlsten M, Andersson S, Bjorklund A, Bjorkstrom NK, Baumann BC, Fauriat C, Alici E, Dilber MS, and Ljunggren HG: NK cell-mediated targeting of human cancer and possibilities for new means of immunotherapy. Cancer Immunol Immunother 57: 1541-1552, 2008.

2 Samara P, Skopeliti M, Tsiatas ML, Georgaki S, Gouloumis C, Voelter W, Dimopoulos AM, Bamias A and Tsitsilonis OE: A cytokine cocktail augments the efficacy of adoptive NK-92 Cell therapy against mouse xenografts of human cancer. Anticancer Res 36: 3373-3382, 2016.

3 Klingemann H: Challenges of cancer therapy with natural killer cells. Cytotherapy 17: 245-249, 2015.

4 Denman CJ, Senyukov VV, Somanchi SS, Phatarpekar PV, Kopp LM, Johnson JL, Singh H, Hurton L, Maiti SN, Huls MH, Champlin RE, Cooper LJ and Lee DA: Membrane-bound IL-21 promotes sustained ex vivo proliferation of human natural killer cells. PloS one 7: e30264, 2012.

5 Fujisaki H, Kakuda H, Shimasaki N, Imai C, Ma J, Lockey T, Eldridge P, Leung WH and Campana D: Expansion of highly cytotoxic human natural killer cells for cancer cell therapy. Cancer Res 69: 4010-4017, 2009.

6 Granzin M, Soltenborn S, Muller S, Kollet J, Berg M, Cerwenka A, Childs RW and Huppert V: Fully automated expansion and activation of clinical-grade natural killer cells for adoptive immunotherapy. Cytotherapy 17: 621-632, 2015.

7 Granzin M, Wagner J, Kohl U, Cerwenka A, Huppert V and Ullrich E: Shaping of natural killer cell antitumor activity by $e x$ vivo cultivation. Front Immunol 8: 458, 2017.

8 Harada H, Saijo K, Watanabe S, Tsuboi K, Nose T, Ishiwata I and Ohno T: Selective expansion of human natural killer cells from peripheral blood mononuclear cells by the cell line, HFWT. Jpn J Cancer Res 93: 313-319, 2002.
9 Torelli GF, Guarini A, Maggio R, Alfieri C, Vitale A and Foa R: Expansion of natural killer cells with lytic activity against autologous blasts from adult and pediatric acute lymphoid leukemia patients in complete hematologic remission. Haematologica 90: 785-792, 2005.

10 Bae DS and Lee JK: Development of NK cell expansion methods using feeder cells from human myelogenous leukemia cell line. Blood Res 49: 154-161, 2014.

11 Phan MT, Lee SH, Kim SK and Cho D: Expansion of NK Cells Using Genetically Engineered K562 Feeder Cells. Methods Mol Biol 1441: 167-174, 2016.

12 Roy A, Krzykwa E, Lemieux R and Neron S: Increased efficiency of gamma-irradiated versus mitomycin C-treated feeder cells for the expansion of normal human cells in longterm cultures. J Hematother Stem Cell Res 10: 873-880, 2001.

13 Lapteva N, Durett AG, Sun J, Rollins LA, Huye LL, Fang J, Dandekar V, Mei Z, Jackson K, Vera J, Ando J, Ngo MC, Coustan-Smith E, Campana D, Szmania S, Garg T, Moreno-Bost A, Vanrhee F, Gee AP and Rooney CM: Large-scale ex vivo expansion and characterization of natural killer cells for clinical applications. Cytotherapy 14: 1131-1143, 2012.

14 Baek HJ, Kim JS, Yoon M, Lee JJ, Shin MG, Ryang DW, Kook $\mathrm{H}$, Kim SK and Cho D: Ex vivo expansion of natural killer cells using cryopreserved irradiated feeder cells. Anticancer Res 33: 2011-2019, 2013.

15 Friedman EJ: Immune modulation by ionizing radiation and its implications for cancer immunotherapy. Curr Pharm Des 8: 1765-1780, 2002.

16 Kim JY, Son YO, Park SW, Bae JH, Chung JS, Kim HH, Chung BS, Kim SH and Kang CD: Increase of NKG2D ligands and sensitivity to NK cell-mediated cytotoxicity of tumor cells by heat shock and ionizing radiation. Exp Mol Med 38: 474-484, 2006. 